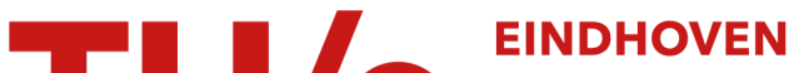 \\ UNIVERSITY OF \\ TECHNOLOGY
}

\section{van der Waals Universality near a Quantum Tricritical Point}

Citation for published version (APA):

Mestrom, P. M. A., Colussi, V. E., Secker, T., Groeneveld, G. P., \& Kokkelmans, S. J. J. M. F. (2020). van der Waals Universality near a Quantum Tricritical Point. Physical Review Letters, 124(14), [143401].

https://doi.org/10.1103/PhysRevLett.124.143401

DOI:

10.1103/PhysRevLett.124.143401

Document status and date:

Published: 08/04/2020

\section{Document Version:}

Publisher's PDF, also known as Version of Record (includes final page, issue and volume numbers)

\section{Please check the document version of this publication:}

- A submitted manuscript is the version of the article upon submission and before peer-review. There can be important differences between the submitted version and the official published version of record. People interested in the research are advised to contact the author for the final version of the publication, or visit the $\mathrm{DOI}$ to the publisher's website.

- The final author version and the galley proof are versions of the publication after peer review.

- The final published version features the final layout of the paper including the volume, issue and page numbers.

Link to publication

\section{General rights}

Copyright and moral rights for the publications made accessible in the public portal are retained by the authors and/or other copyright owners and it is a condition of accessing publications that users recognise and abide by the legal requirements associated with these rights.

- Users may download and print one copy of any publication from the public portal for the purpose of private study or research.

- You may not further distribute the material or use it for any profit-making activity or commercial gain

- You may freely distribute the URL identifying the publication in the public portal.

If the publication is distributed under the terms of Article 25fa of the Dutch Copyright Act, indicated by the "Taverne" license above, please follow below link for the End User Agreement:

www.tue.nl/taverne

Take down policy

If you believe that this document breaches copyright please contact us at:

openaccess@tue.nl

providing details and we will investigate your claim. 


\title{
van der Waals Universality near a Quantum Tricritical Point
}

\author{
P. M. A. Mestrom๑, V. E. Colussi®, T. Secker, G. P. Groeneveld๑, and S. J. J. M. F. Kokkelmans $\odot$ \\ Eindhoven University of Technology, P. O. Box 513, 5600 MB Eindhoven, Netherlands
}

(Received 23 December 2019; accepted 10 March 2020; published 8 April 2020)

\begin{abstract}
We study the three-body scattering hypervolume $D$ of atoms whose scattering length $a$ is on the order of or smaller than the typical range $r_{\mathrm{vdW}}$ of the van der Waals attraction. We find that the real part of $D$ behaves universally in this weakly interacting regime $\left(|a| / r_{\mathrm{vdW}} \lesssim 1\right)$ in the absence of trimer resonances. This universality originates from hard-spherelike collisions that dominate elastic three-body scattering. We use this result to make quantitative predictions for the thermodynamics and elementary excitations of an atomic Bose-Einstein condensate in the vicinity of a quantum tricritical point, including quantum droplets stabilized by effective three-body interactions.
\end{abstract}

DOI: $10.1103 /$ PhysRevLett.124.143401

Introduction.-The thermodynamics of ultracold dilute quantum gases is determined by the asymptotic behavior of few-body scattering processes. Bosonic many-body systems can be universally described with only one parameter to characterize the two-body interactions: the $s$-wave scattering length $a[1]$. However, even in the limit $a \rightarrow 0$ when twobody interactions effectively vanish, system properties are still determined by few-body scattering. The quantum phase diagram of a uniform Bose-Einstein condensate (BEC) in this limit is determined by the three-body scattering hypervolume $D$ that is the analog of $a[2,3]$. Provided $\operatorname{Re}(D)>0$, a quantum tricritical point exists at $a=0$ where the boundaries between liquid and gaseous ground states and vacuum meet [3]. Quantum tricritical points are rare, occurring for certain metallic magnets [4-6] and for a generalized Dicke model [7].

The ground-state energy density $\mathcal{E}$ of a uniform BEC at $a=0$ is determined by $D$ via

$$
\mathcal{E}(n)=\frac{\hbar^{2} D n^{3}}{6 m}+\cdots,
$$

where the dots indicate terms with higher powers of the number density $n$ [2]. In the liquid phase when $a<0$ and $\operatorname{Re}(D)>0$, effective three-body repulsion and two-body attraction compete. This provides a three-body stabilization mechanism for liquid quantum droplets against collapse [8]. In Ref. [9], it was suggested that the experimentally observed collapse [10-13] is a relaxation process towards the liquid equilibrium state. Recently, quantum droplets were predicted [14] and experimentally observed in both mixed [15-17] and dipolar BECs [18-20], however, these systems were based on two-body stabilization mechanisms. Additionally, in typical alkali systems, $D$ acquires an imaginary part proportional to the three-body recombination rate [21,22], so that these states are inherently metastable. Ultimately, a quantitative understanding of droplet properties near the quantum tricritical point depends on the sign and magnitude of $\operatorname{Re}(D)$ for realistic systems.
For weakly interacting systems, Refs. [2,3,21,23] investigated the scattering hypervolume, demonstrating how $D$ is influenced by nonuniversal three-body quasibound states $[21,23]$ and is connected to physical observables at $a=0$ [3]. Nevertheless, none of these studies solved the threebody problem for two-body interaction models that contain the long-range atomic van der Waals attraction. We find that this is the essential ingredient for quantitative predictions of $D$, whose real part is universally fixed by the van der Waals range $r_{\mathrm{vdW}}=\left(m C_{6} / \hbar^{2}\right)^{1 / 4} / 2$, where $C_{6}$ is the dispersion coefficient describing the long-range behavior of the interatomic interaction. Although the mechanisms differ, van der Waals universality also determines $D$ in the strongly interacting regime $\left(|a| / r_{\mathrm{vdW}} \gg 1\right)$ by setting the spectrum of Efimov trimers [24-35].

In this Letter, we present a numerical study of the scattering hypervolume for identical bosons interacting via pairwise van der Waals potentials in the weakly interacting regime $\left(|a| \lesssim r_{\mathrm{vdW}}\right)$. We find that $\operatorname{Re}(D)$ is predominantly determined by $a$ and $r_{\mathrm{vdW}}$ and analyze the origin of this van der Waals universality. This is used to make universal quantitative predictions for atomic BECs near the quantum tricritical point, including quantum droplets stabilized by effective three-body interactions.

Method.-Here we use the Alt, Grassberger, and Sandhas (AGS) approach [36] which has been proven to be a powerful method for calculating the scattering hypervolume [23]. The AGS equations,

$$
\begin{aligned}
& U_{00}(z)=\sum_{\alpha=1}^{3} T_{\alpha}(z) G_{0}(z) U_{\alpha 0}(z) \\
& U_{\alpha 0}(z)=G_{0}^{-1}(z)+\sum_{\substack{\beta=1 \\
\beta \neq \alpha}}^{3} T_{\beta}(z) G_{0}(z) U_{\beta 0}(z)
\end{aligned}
$$$$
\text { for } \alpha=1,2,3 \text {, }
$$ 
are Faddeev equations for the three-body transition operators $U_{\alpha \beta}(z)$, where $z$ denotes the three-body energy. The index $\alpha(\beta)$ labels the partitions for the outgoing (incoming) state which consists of three free particles $(\alpha=0)$ or a free particle and dimer $(\alpha=1,2,3) . G_{0}(z)$ is the free resolvent $\left(z-H_{0}\right)^{-1}$, where $H_{0}$ is the three-body kinetic energy operator for the relative motion. $T_{\alpha}(z)$ represents the twoparticle transition operator for the pair $\beta \gamma(\beta, \gamma=1,2,3$, $\beta \neq \gamma \neq \alpha)$, i.e., $T_{\alpha}(z)=V_{\beta \gamma}+V_{\beta \gamma} G_{0}(z) T_{\alpha}(z)$, where $V_{\beta \gamma}$ is the interaction potential acting within the pair $\beta \gamma$. From the transition amplitude corresponding to $U_{00}(0)$, we determine the scattering hypervolume in the same way as described by Ref. [23] which adopts the Weinberg expansion for $T_{\alpha}(z)[37,38]$.

In the present study, we analyze the scattering hypervolume for identical bosons that interact via various van der Waals potentials, indicated by $V_{\mathrm{LJ}}, V_{\text {zero }}, V_{\text {exp }}$, and $V_{\mathrm{sc}}$. Their long-range behavior is described by the van der Waals tail $-C_{6} / r^{6}$, but their short-range behavior is completely different. Here $V_{\mathrm{LJ}}$ is the Lennard-Jones potential

$$
V_{\mathrm{LJ}}=-\frac{C_{6}}{r^{6}}\left(1-\frac{\lambda^{6}}{r^{6}}\right)
$$

where $\lambda$ locates the potential barrier. The formulas for the other potentials can be found in the Supplemental Material [39]. By adjusting the potential depths, we tune the scattering length $a$ and the number of two-body bound states [40]. We indicate the number of $s$-wave dimer states by adding an additional index to the potential name. For example, $V_{\mathrm{LJ}}^{(1)}$ supports one $s$-wave dimer state.

van der Waals universality.-Figure 1 shows a comparison of $D$ in the weakly interacting regime for multiple van der Waals potentials. Despite the presence of several three-body resonances, $\operatorname{Re}(D)$ behaves universally in contrast to $\operatorname{Im}(D)$. Physically, $\operatorname{Im}(D)$ is determined by recombination pathways where three atoms approach at short distances, whereas $\operatorname{Re}(D)$ is determined through many competing pathways for elastic scattering at different length scales. When the long-range pathways dominate, $\operatorname{Re}(D)$ is set by the van der Waals tail and the asymptotics of the twobody scattering wave function characterized by $r_{\mathrm{vdW}}$ and $a$, respectively. We note that this picture only applies in the absence of a three-body resonance where the universality in $\operatorname{Re}(D)$ can be broken as shown in Fig. 1.

To identify the dominant pathway for elastic three-body scattering, we study the scaling behavior of $\operatorname{Re}(D)$. In the strongly interacting regime $\left(|a| / r_{\mathrm{vdW}} \gg 1\right)$, pathways that involve a single reflection from the three-body effective potentials contribute to $D$ as $1689 a^{4}$ [23,27], both for positive and negative scattering lengths. This reflection occurs off a barrier in the three-body effective potential which acts as a hard hypersphere of hyperradius $|a|$. A similar result $D=1761.5 a^{4}$ is found for bosons interacting pairwise via a hard-sphere potential [2], whose repulsive

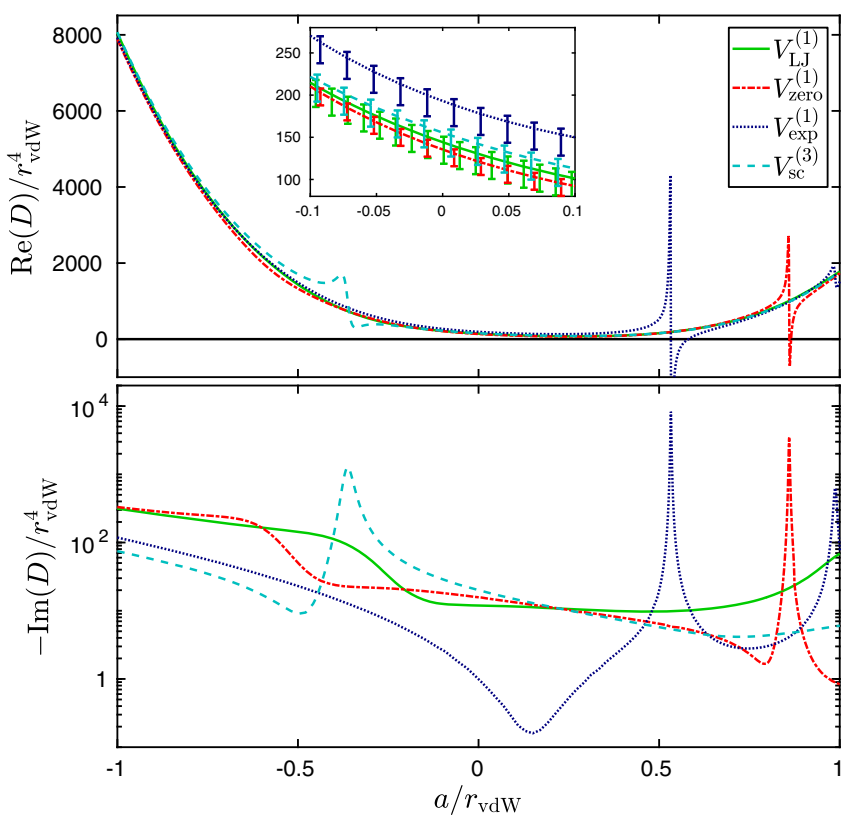

FIG. 1 . Three-body scattering hypervolume $D$ corresponding to multiple van der Waals potentials as a function of the two-body scattering length $a$ [41]. The inset shows the real part of $D$ near the zero crossing of $a$.

character makes it inherently different from the attractive potentials considered in this Letter. However, in the weakly interacting regime $\left(|a| / r_{\mathrm{vdW}} \lesssim 1\right)$, the scattering length cannot be the only length scale that determines the location of the barrier. Therefore we generalize the hard-hypersphere radius $R_{\mathrm{hh}}$ in the hard-hypersphere formula,

$$
\operatorname{Re}(D)=1689 R_{\mathrm{hh}}^{4}
$$

to $\left|a-a_{\mathrm{hh}}^{ \pm}\right|$, where the offsets $a_{\mathrm{hh}}^{ \pm}$capture finite-range effects. We choose $a_{\mathrm{hh}}^{ \pm}$such that the hard-hypersphere formula matches the value of $\operatorname{Re}(D)$ at $a / r_{\mathrm{vdW}}= \pm 1$. This results in the universal values $a_{\mathrm{hh}}^{+} / r_{\mathrm{vdW}}=-0.010(3)$ and $a_{\mathrm{hh}}^{-} / r_{\mathrm{vdW}}=0.474(7)$ for which the uncertainties are estimated by the deviation among the considered potentials. That Eq. (4) with $R_{\mathrm{hh}}=\left|a-a_{\mathrm{hh}}^{ \pm}\right|$describes $\operatorname{Re}(D)$ over a range of scattering lengths as shown in Fig. 2 confirms the dominance of hard-hyperspherelike collisions in the weakly interacting regime, except in the crossover regime $-0.1 \lesssim a / r_{\mathrm{vdW}} \lesssim 0.6$.

As long as $R_{\mathrm{hh}}>\lambda$, where $\lambda$ is a characteristic length scale of the short-range details of the interaction potential, one can expect that $R_{\mathrm{hh}}$ is set by $a$ and $r_{\mathrm{vdW}}$. Figure 2 shows that the regime in which $\left|a-a_{\mathrm{hh}}^{ \pm}\right|$does not describe $R_{\mathrm{hh}}$ is roughly determined by $R_{\mathrm{hh}} \lesssim \lambda$. In the limit of deep van der Waals potentials, $\lambda / r_{\mathrm{vdW}}$ approaches zero. This implies that the $-C_{6} / r^{6}$ behavior is approached at smaller values of $r / r_{\text {vdw }}$. Therefore, we expect that $\operatorname{Re}(D)$ of deep van der Waals potentials behaves universally in the complete 


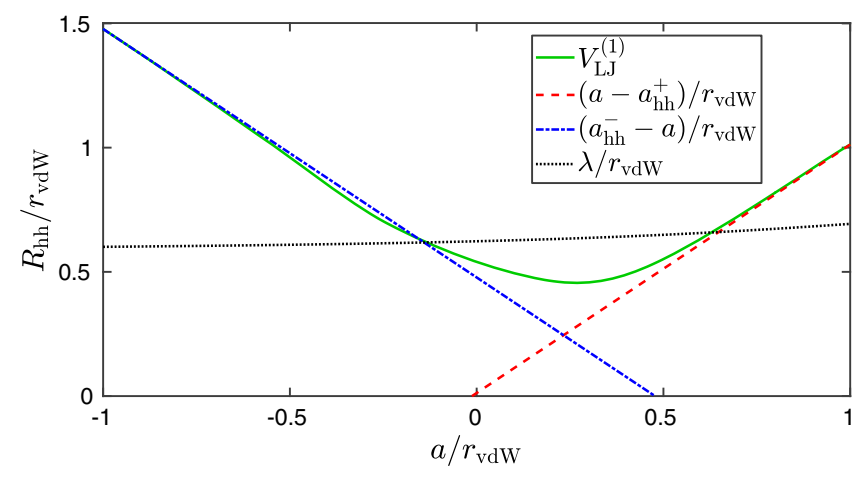

FIG. 2. The hard-sphere radius $R_{\mathrm{hh}}=[\operatorname{Re}(D) / 1689]^{1 / 4}$ corresponding to the potential $V_{\mathrm{LJ}}^{(1)}$ (green solid line) as a function of the two-body scattering length $a$. The dashed and dash-dotted curves show $\left(a-a_{\mathrm{hh}}^{+}\right) / r_{\mathrm{vdW}}$ and $\left(a_{\mathrm{hh}}^{-}-a\right) / r_{\mathrm{vdW}}$, respectively, for which $a_{\mathrm{hh}}^{ \pm}$is modified to match $\operatorname{Re}(D)$ at $a / r_{\mathrm{vdW}}= \pm 1$. The values of $a_{\mathrm{hh}}^{+} / r_{\mathrm{vdW}}$ and $a_{\mathrm{hh}}^{-} / r_{\mathrm{vdW}}$ are -0.012 and 0.477 , respectively. The dotted curve displays the position $\lambda / r_{\mathrm{vdW}}$ of the repulsive barrier of $V_{\mathrm{LJ}}^{(1)}$. Note that $R_{\mathrm{hh}}$ is slightly affected by a trimer resonance near $a / r_{\mathrm{vdW}} \simeq-0.3$ [39].

weakly interacting regime in the absence of trimer resonances [42].

For a Lennard-Jones potential supporting no two-body bound states $\left(V_{\mathrm{LJ}}^{(0)}\right)$, we find $D / r_{\mathrm{vdW}}^{4}=86 \pm 2$ at $a=0$ and compare this result to the value 90 predicted by Zwerger $[3,39,43]$. This prediction is based upon an earlier manybody calculation for the energy per particle of a Bose fluid at zero temperature and zero pressure near the quantum tricritical point [44]. This close agreement demonstrates that $D$ can be determined from properties of ultracold Bose systems near the quantum tricritical point, which we turn to presently.

Thermodynamics and elementary excitations.-How does the scattering hypervolume determine the groundstate properties and excitations of a BEC near the quantum tricritical point? To understand these effects, we follow Ref. [3] and study the effective Lagrangian density valid at weak interactions and zero temperature,

$$
\begin{aligned}
\mathcal{L}= & \frac{i \hbar}{2}\left[\Psi^{*} \dot{\Psi}-\Psi \dot{\Psi}^{*}\right]-\frac{\hbar^{2}}{2 m}|\nabla \Psi|^{2}-V_{\mathrm{ext}}(\mathbf{r})|\Psi|^{2} \\
& -\frac{2 \pi \hbar^{2} a}{m}|\Psi|^{4}-\frac{\hbar^{2} D}{6 m}|\Psi|^{6} .
\end{aligned}
$$

The Gross-Pitaevskii equation follows from minimizing the action $S=\int d^{3} r d t \mathcal{L}$, giving

$i \hbar \dot{\Psi}=-\frac{\hbar^{2}}{2 m} \nabla^{2} \Psi+V_{\text {ext }}(\mathbf{r}) \Psi+\frac{4 \pi \hbar^{2} a}{m}|\Psi|^{2} \Psi+\frac{\hbar^{2} D}{2 m}|\Psi|^{4} \Psi$.

The condensate wave function is formulated as $\Psi(\mathbf{r}, t)=$ $\sqrt{n(\mathbf{r}, t)} e^{-i \phi(\mathbf{r}, t)}$ satisfying the Josephson relation $\hbar \dot{\phi}=-\mu$ under stationary conditions. In Eq. (6), higher-order effects have been ignored as well as the energy dependence of the few-body scattering amplitudes [45-47]. In the following, we focus on signatures of $D$ in uniform and trapped systems with $V_{\text {ext }}(\mathbf{r})=m \omega_{\mathrm{ho}}^{2}\left(\lambda_{x}^{2} x^{2}+\lambda_{y}^{2} y^{2}+\lambda_{z}^{2} z^{2}\right) / 2$. We define the oscillator length $l_{\text {ho }}=\sqrt{\hbar / m \omega_{\text {ho }}}$ and the geometric means $\bar{\omega}=\omega_{\text {ho }}\left(\lambda_{x} \lambda_{y} \lambda_{z}\right)^{1 / 3}$ and $\bar{l}=\sqrt{\hbar / m \bar{\omega}}$. In this analysis, we neglect the imaginary part of $D$ since our results for $V_{\mathrm{LJ}}^{(1)}$ show that $|\operatorname{Re}(D) / \operatorname{Im}(D)|$ varies from roughly 10 near $a=0$ to roughly 25 near $a / r_{\mathrm{vdW}}= \pm 1$, and we return to this point below when discussing the experimental outlook.

At the point $a=0$, where we estimate $\operatorname{Re}(D) / r_{\mathrm{vdW}}^{4} \approx$ 100, Eqs. (5) and (6) contain only effective three-body interactions. This regime for a uniform gas was considered in Ref. [3], finding chemical potential $\mu=D \hbar^{2} n^{2} / 2 m$, pressure $P=\left(8 m / 9 D \hbar^{2}\right)^{1 / 2} \mu^{3 / 2}$ and sound velocity $c=$ $\hbar n \sqrt{D} / m$. For the ground state of a trapped gas, the ThomasFermi approximation gives $n(\mathbf{r})=\left(2 m\left[\mu-V_{\text {ext }}(\mathbf{r})\right] /\right.$ $\left.D \hbar^{2}\right)^{1 / 2}$. After normalization, the chemical potential is fixed to $\mu / \hbar \bar{\omega}=\zeta^{1 / 4} / \pi$ in terms of the three-body Thomas-Fermi parameter $\zeta=D N^{2} / \bar{l}^{4}$. Likewise, integrating the thermodynamic relation $\mu=\partial_{N} E$ gives energy per particle $E / N=2 \mu / 3$, from which we infer the interaction energy per particle $E_{\text {int }} / N=\mu / 6$ as a consequence of the virial theorem [48]. At the cloud boundaries $\mu=m \omega_{\mathrm{ho}}^{2} \lambda_{\eta}^{2} R_{\eta}^{2} / 2(\eta=x, y, z)$, and we estimate the spatial extent of the cloud from the geometric mean of the semiaxes $\bar{R} \equiv\left(R_{x} R_{y} R_{z}\right)^{1 / 3}=\sqrt{2 / \pi} \bar{l} \zeta^{1 / 8}$. Compared to the Thomas-Fermi limit for two-body interactions, we find that a smaller portion of the total energy is involved in interactions, however, due to the $N^{2}$ scaling of $\zeta$, all energies and radii scale with higher powers of $N$.

To investigate the shift of discretized collective modes in a harmonic trap at $a=0$, we use a time-dependent trial wave function [49-51]

$$
\Psi(x, y, z, t)=A(t) \prod_{\eta=x, y, z} e^{-\left[\left(\eta-\eta_{0}\right)^{2} / 2 w_{\eta}^{2}\right]+i \eta \alpha_{\eta}+i \eta^{2} \beta_{\eta}},
$$

with time-dependent variational parameters $\left\{w_{\eta}, \eta_{0}, \alpha_{\eta}\right.$, $\left.\beta_{\eta}\right\}_{\eta=x, y, z}$. The magnitude of $A$ is set by particle number conservation. Minimizing the action with respect to the variational parameters, we find

$$
\begin{gathered}
\partial_{\tau}^{2} \eta_{0}+\lambda_{\eta}^{2} \eta_{0}=0, \\
\partial_{\tau}^{2} v_{\eta}+\lambda_{\eta}^{2} v_{\eta}=\frac{1}{v_{\eta}^{3}}+\frac{K}{v_{\eta}\left(v_{x} v_{y} v_{z}\right)^{2}} \quad(\eta=x, y, z),
\end{gathered}
$$

with $K=2 D N^{2} / 9 \sqrt{3} \pi^{3} l_{\text {ho }}^{4}$ and dimensionless scalings $v_{\eta}=w_{\eta} / l_{\mathrm{ho}}$ and $\tau=\omega_{\mathrm{ho}} t$. Equation (8) describes dipole oscillations of the condensate center with trap frequencies 

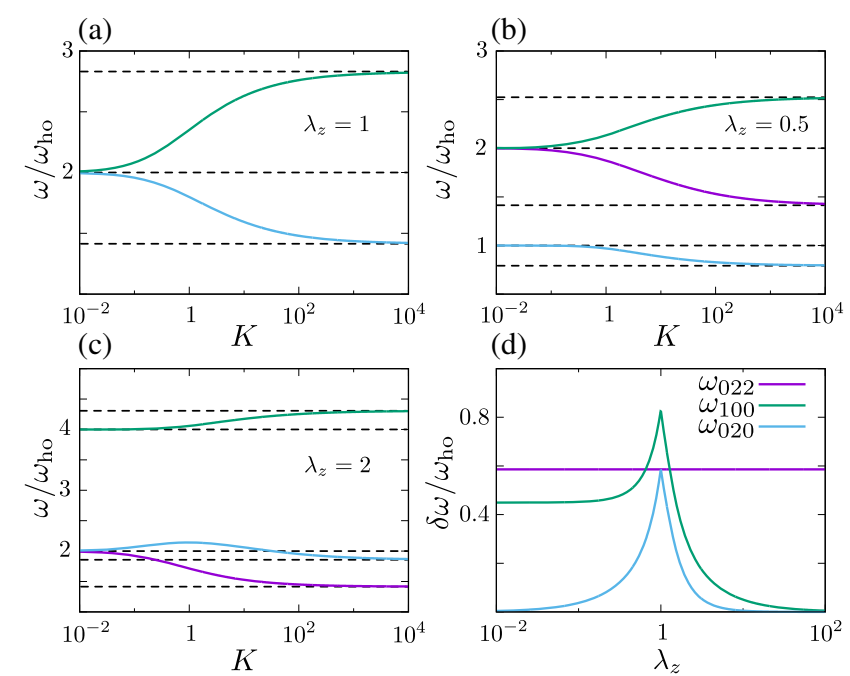

FIG. 3. Collective mode frequencies at $a=0$ versus $K=$ $2 D N^{2} / 9 \sqrt{3} \pi^{3} l_{\text {ho }}^{4}$ for (a) isotropic, (b) cigar, and (c) pancake geometries. We set $\lambda_{x}=\lambda_{y}=1$ and vary $\lambda_{z}$. The $\omega_{020}$ and $\omega_{022}$ modes are degenerate for an isotropic trap. Dashed lines indicate results in the noninteracting and Thomas-Fermi limits. (d) Contrast $\left.\delta \omega_{n l m} \equiv \omega_{n l m}\right|_{K \gg 1}-\left.\omega_{n l m}\right|_{K=0}$ versus trap aspect ratio $\lambda_{z}$.

in agreement with the Kohn theorem [52]. Linearizing Eq. (9) about equilibrium, yields mode frequencies $\omega_{022}, \omega_{100}$, and $\omega_{020}$ in terms of principle and angular quantum numbers parametrized as $\omega_{n l m}$ [49] (see Ref. [39]). Our results for these modes at $a=0$ are shown in Figs. 3(a)-3(d) over a range of geometries and values of $K$ compared against results in the noninteracting and Thomas-Fermi limits. Experimentally, $D$ can be inferred from frequency shifts intermediate to these limits. From Fig. 3(d), this contrast is maximized for the breathing mode $\omega_{100}$ in an isotropic geometry. In the Thomas-Fermi limit, this mode shifts to $2 \sqrt{2} \omega_{\text {ho }}$, which is beyond the result $\sqrt{5} \omega_{\text {ho }}$ for two-body interactions [53]. Physically, the simultaneous compression of this mode along all axes leads to increased densities near trap center and the largest nonlinear interaction effects [54].

For $a<0$, it is possible that $D>0$ can stabilize the BEC against collapse. Taking the Gaussian trial wave function of Eq. (7) with $\eta_{0}=\alpha_{\eta}=\beta_{\eta}=0$ and $w_{\eta}=w$, we minimize the energy

$$
E=\frac{3 \hbar^{2}}{4 m} \frac{N}{w^{2}}+\frac{\hbar^{2} a}{\sqrt{2 \pi} m} \frac{N^{2}}{w^{3}}+\frac{\hbar^{2} D}{18 \sqrt{3} \pi^{3} m} \frac{N^{3}}{w^{6}}
$$

in the absence of a trapping potential for a fixed number of particles $N$. We find that no droplets exist for $N \leq N_{\mathrm{c}}$, where

$$
N_{\mathrm{c}}=\frac{2^{7 / 2}}{3^{11 / 4} \sqrt{\pi}} \frac{\sqrt{D}}{a^{2}} .
$$

The droplets are metastable for $N_{\mathrm{c}}<N \leq 3 \sqrt{3} N_{\mathrm{c}} / 4=N_{\mathrm{s}}$ and stable for $N>N_{\mathrm{s}}$. The variational dependence of the (a)

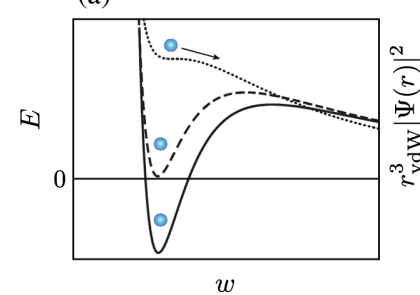

(c)

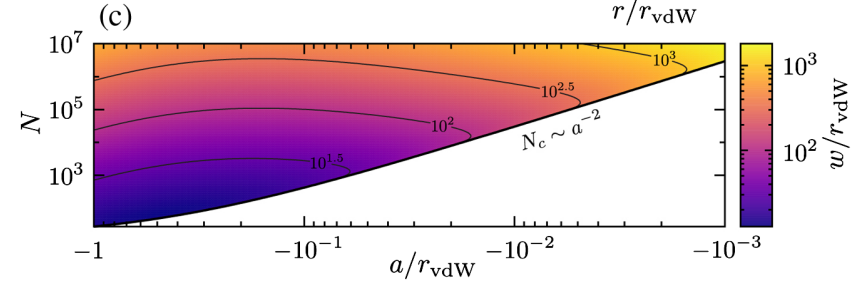

FIG. 4. Ground-state properties of Bose droplets. (a) Illustrated dependence of the energy $E$ given by Eq. (10) with width $w$ for $N>N_{\mathrm{s}}$ (solid), $N=N_{\mathrm{s}}$ (dashed), and $N=N_{\mathrm{c}}$ (dotted). (b) Numerical ground-state profile versus radial coordinate for $N / N_{\mathrm{s}} \approx 1$ (dotted), 10 (dash-dotted), 100 (dashed), and 1000 (solid) evaluated at $a=-0.08 r_{\mathrm{vdW}}$ taking our result for $V_{\mathrm{LJ}}^{(1)}$, $\operatorname{Re}(D)=197 r_{\text {vdW }}^{4}$ (see Fig. 1 inset), compared with the large- $N$ density $n_{0}$ indicated by the solid red curve. (c) Droplet width $w$ versus $a$ and $N$ from the Gaussian trial wave function Eq. (7) using Eq. (4) with $R_{\mathrm{hh}}=\left|a-0.477 r_{\mathrm{vdw}}\right|$ and neglecting $\operatorname{Im}(D)$. The liquid-to-gas transition at $N_{\mathrm{c}}$ is indicated by the solid line.

energy as a function of the width $w$ is illustrated in Fig. 4(a) for the unstable, metastable, and stable regimes. The density profiles of the droplets numerically obtained from Eq. (6) are depicted in Fig. 4(b) for various $N$ which shows that the Gaussian trial wave function is reasonable near the metastable regime. Figure 4(c) shows the phase diagram of the Bose fluid for $a<0$ using Eq. (4) with $R_{\mathrm{hh}}=\mid a-$ $0.477 r_{\mathrm{vdW}} \mid$ while neglecting $\operatorname{Im}(D)$. For large $N$ the density profile is almost constant with density $n_{0}=6 \pi|a| / D$ [8], which is approximately a factor 2.18 larger than the center density of a droplet with $N=N_{\mathrm{c}}$. In the large $N$ limit, the Gaussian trial wave function overestimates the center density by roughly a factor 1.84 . Previous estimates of $n_{0}$ in Ref. [9] are larger by roughly a factor of 5 to 10 than our result, which we attribute to an underestimation of effective three-body repulsion in that work.

Experimental outlook.-Let us now discuss the experimental possibilities to observe the effects of $D$. For this purpose, we take $\operatorname{Im}(D)$ into account in the typical lifetime $\tau_{\text {life }}=1 / L_{3} n^{2}$, where $L_{3}=-\operatorname{Im}(D) \hbar / m$ determines the loss rate of atoms from the BEC via three-body recombination [21]. For quantum droplets with density $n_{0}$ and chemical potential $\mu_{0}=-6 \pi^{2} \hbar^{2} a^{2} / m \operatorname{Re}(D)$ [8], we compare $\tau_{\text {life }}$ to the characteristic timescale $\tau_{0}=\hbar /\left|\mu_{0}\right|$ and find $\tau_{\text {life }} / \tau_{0}=-\operatorname{Re}(D) / 6 \operatorname{Im}(D)$, which is typically larger than one according to our results for $D$. Since $\tau_{\text {life }} \propto[\operatorname{Re}(D)]^{2} / \operatorname{Im}(D) a^{2}$, longer lifetimes can be achieved at smaller $|a|$. However, the critical number $N_{\mathrm{c}}$ also increases in this limit [see Fig. 4(c)]. 
To observe the collective mode frequencies at $a=0$, we require $\tau_{\text {life }}>\tau_{\text {ho }}=2 \pi / \omega_{\mathrm{ho}}$. In the Thomas-Fermi limit, we find

$$
\frac{\tau_{\text {life }}}{\tau_{\text {ho }}}=-\frac{\operatorname{Re}(D)}{\operatorname{Im}(D)} \frac{1}{2 \times 3^{5 / 8}(2 \pi)^{3 / 4} \lambda_{z}^{1 / 2} K^{1 / 4}}
$$

for cylindrically symmetric traps with $\lambda_{x}=\lambda_{y}=1 \mathrm{using}$ the center density to estimate $\tau_{\text {life }}$. In general, smaller $\lambda_{z}$ is advantageous for achieving large $K$ for fixed $\tau_{\text {life }} / \tau_{\text {ho }}$ and $D$. Figure $3(\mathrm{~d})$ shows that $\delta \omega_{022} / \omega_{\text {ho }}$ and $\delta \omega_{100} / \omega_{\text {ho }}$ are roughly 0.5 for small $\lambda_{z}$ which makes these modes suitable for extracting $\operatorname{Re}(D)$ from the $K$ dependence of the corresponding mode frequencies. Cigar-shaped traps can also be used to measure the speed of sound at $a=0$ provided that the characteristic distance $c \tau_{\text {life }}$ is large enough to resolve experimentally. Using our previous estimate for $\tau_{\text {life }}$ in the Thomas-Fermi limit, we find

$$
c \tau_{\text {life }}=-\sqrt{\frac{\pi}{2}} \frac{[\operatorname{Re}(D)]^{5 / 4}}{\operatorname{Im}(D)} \frac{\sqrt{N}}{\zeta^{3 / 8}},
$$

which scales as $\lambda_{z}^{-1 / 4}$.

Conclusion.-We study the scattering hypervolume $D$ for identical bosons interacting via pairwise van der Waals potentials. Our results show that $\operatorname{Re}(D)$ is predominantly determined by the long-range two-body properties $r_{\mathrm{vdW}}$ and $a$. The van der Waals universality of this behavior is due to dominant hard-hypersphere scattering. However, $\operatorname{Im}(D)$ depends strongly on the short-range details of the interaction, resulting in nonuniversal behavior. In the limit of vanishing $a, \operatorname{Re}(D)$ determines the quantum phase diagram near the tricritical point. Using the van der Waals universality of $\operatorname{Re}(D)$, we make quantitative predictions for the properties of atomic BECs, including the formation of quantum droplets.

Further studies of $D$ for deeper van der Waals potentials need to be conducted to test the robustness of the universal behavior in $\operatorname{Re}(D)$ in the weakly interacting regime. The influence of multichannel physics on $D$ could lead to new interesting phenomena at small scattering lengths due to additional parameters characterizing the zero crossing of $a$ [55-57]. Dynamical studies including effective three-body repulsion are needed to understand droplet formation.

We thank Wilhelm Zwerger, Jinglun Li, Silvia Musolino, and Denise Ahmed-Braun for fruitful discussions. This research is financially supported by the Netherlands Organisation for Scientific Research (NWO) under Grant No. 680-47-623 and by the Foundation for Fundamental Research on Matter (FOM).

* Corresponding author. p.m.a.mestrom@tue.nl

[1] K. Huang, Statistical Mechanics (Wiley, New York, 1963).
[2] S. Tan, Phys. Rev. A 78, 013636 (2008).

[3] W. Zwerger, J. Stat. Mech. (2019) 103104.

[4] D. Belitz and T. R. Kirkpatrick, Phys. Rev. Lett. 119, 267202 (2017).

[5] S. Friedemann, W. J. Duncan, M. Hirschberger, T. W. Bauer, R. Küchler, A. Neubauer, M. Brando, C. Pfleiderer, and F. M. Grosche, Nat. Phys. 14, 62 (2018).

[6] U.S. Kaluarachchi, V. Taufour, S. L. Bud'ko, and P. C. Canfield, Phys. Rev. B 97, 045139 (2018).

[7] Y. Xu and H. Pu, Phys. Rev. Lett. 122, 193201 (2019).

[8] A. Bulgac, Phys. Rev. Lett. 89, 050402 (2002).

[9] B. Gao, J. Phys. B 37, L227 (2004).

[10] J. M. Gerton, D. Strekalov, I. Prodan, and R. G. Hulet, Nature (London) 408, 692 (2000).

[11] J. L. Roberts, N. R. Claussen, S. L. Cornish, E. A. Donley, E. A. Cornell, and C. E. Wieman, Phys. Rev. Lett. 86, 4211 (2001).

[12] E. A. Donley, N. R. Claussen, S. L. Cornish, J. L. Roberts, E. A. Cornell, and C. E. Wieman, Nature (London) 412, 295 (2001).

[13] C. Eigen, A. L. Gaunt, A. Suleymanzade, N. Navon, Z. Hadzibabic, and R. P. Smith, Phys. Rev. X 6, 041058 (2016).

[14] D. S. Petrov, Phys. Rev. Lett. 115, 155302 (2015).

[15] C. R. Cabrera, L. Tanzi, J. Sanz, B. Naylor, P. Thomas, P. Cheiney, and L. Tarruell, Science 359, 301 (2018).

[16] P. Cheiney, C. R. Cabrera, J. Sanz, B. Naylor, L. Tanzi, and L. Tarruell, Phys. Rev. Lett. 120, 135301 (2018).

[17] G. Semeghini, G. Ferioli, L. Masi, C. Mazzinghi, L. Wolswijk, F. Minardi, M. Modugno, G. Modugno, M. Inguscio, and M. Fattori, Phys. Rev. Lett. 120, 235301 (2018).

[18] H. Kadau, M. Schmitt, M. Wenzel, C. Wink, T. Maier, I. Ferrier-Barbut, and T. Pfau, Nature (London) 530, 194 (2016).

[19] I. Ferrier-Barbut, H. Kadau, M. Schmitt, M. Wenzel, and T. Pfau, Phys. Rev. Lett. 116, 215301 (2016).

[20] L. Chomaz, S. Baier, D. Petter, M. J. Mark, F. Wächtler, L. Santos, and F. Ferlaino, Phys. Rev. X 6, 041039 (2016).

[21] S. Zhu and S. Tan, arXiv:1710.04147v1.

[22] E. Braaten and H.-W. Hammer, Phys. Rep. 428, 259 (2006).

[23] P. M. A. Mestrom, V. E. Colussi, T. Secker, and S. J. J. M. F. Kokkelmans, Phys. Rev. A 100, 050702(R) (2019).

[24] M. Berninger, A. Zenesini, B. Huang, W. Harm, H.-C. Nägerl, F. Ferlaino, R. Grimm, P. S. Julienne, and J. M. Hutson, Phys. Rev. Lett. 107, 120401 (2011).

[25] P. Naidon and S. Endo, Rep. Prog. Phys. 80, 056001 (2017).

[26] C. H. Greene, P. Giannakeas, and J. Pérez-Ríos, Rev. Mod. Phys. 89, 035006 (2017).

[27] J. P. D’Incao, J. Phys. B 51, 043001 (2018).

[28] J. Wang, J. P. D'Incao, B. D. Esry, and C. H. Greene, Phys. Rev. Lett. 108, 263001 (2012).

[29] Y. Wang, J. Wang, J. P. D'Incao, and C. H. Greene, Phys. Rev. Lett. 109, 243201 (2012).

[30] R. Schmidt, S. Rath, and W. Zwerger, Eur. Phys. J. B 85, 386 (2012).

[31] P. Naidon, S. Endo, and M. Ueda, Phys. Rev. A 90, 022106 (2014).

[32] D. Blume, Few-Body Syst. 56, 859 (2015).

[33] P. Naidon, E. Hiyama, and M. Ueda, Phys. Rev. A 86, 012502 (2012). 
[34] P. K. Sørensen, D. V. Fedorov, A. S. Jensen, and N. T. Zinner, Phys. Rev. A 88, 042518 (2013).

[35] R. Chapurin, X. Xie, M. J. Van de Graaff, J. S. Popowski, J. P. D'Incao, P. S. Julienne, J. Ye, and E. A. Cornell, Phys. Rev. Lett. 123, 233402 (2019).

[36] E. Alt, P. Grassberger, and W. Sandhas, Nucl. Phys. B2, 167 (1967).

[37] S. Weinberg, Phys. Rev. 131, 440 (1963).

[38] P. M. A. Mestrom, T. Secker, R. M. Kroeze, and S. J. J. M. F. Kokkelmans, Phys. Rev. A 99, 012702 (2019).

[39] See Supplemental Material at http://link.aps.org/ supplemental/10.1103/PhysRevLett.124.143401 for additional details of our calculations and results.

[40] The scattering length contains a resonant and nonresonant contribution. The latter is known as the background scattering length and is on the order of $r_{\mathrm{vdW}}$ for the considered van der Waals potentials.

[41] There could be additional narrow trimer resonances for which our grid for $a$ is too sparse. Such resonances would have a width smaller than $\delta a=10^{-3} r_{\mathrm{vdW}}$.

[42] This expectation is consistent with our results of $\operatorname{Re}(D)$ for the potentials $V_{\mathrm{sc}}^{(1)}, V_{\mathrm{sc}}^{(2)}$, and $V_{\mathrm{sc}}^{(3)}$ which approach the universal curve as the potential depth increases [39].

[43] The uncertainty in this prediction is determined by the validity of the approximations that are made in Ref. [44], including the trial wave function that is used to describe the ground state of the Bose fluid near the quantum tricritical point.
[44] M. D. Miller, L. H. Nosanow, and L. J. Parish, Phys. Rev. B 15, 214 (1977).

[45] H. Fu, Y. Wang, and B. Gao, Phys. Rev. A 67, 053612 (2003).

[46] A. Collin, P. Massignan, and C. J. Pethick, Phys. Rev. A 75, 013615 (2007)

[47] M. Thøgersen, N. T. Zinner, and A. S. Jensen, Phys. Rev. A 80, 043625 (2009).

[48] F. Dalfovo, S. Giorgini, L. P. Pitaevskii, and S. Stringari, Rev. Mod. Phys. 71, 463 (1999).

[49] V. M. Pérez-García, H. Michinel, J. I. Cirac, M. Lewenstein, and P. Zoller, Phys. Rev. Lett. 77, 5320 (1996).

[50] S. Yi and L. You, Phys. Rev. A 63, 053607 (2001).

[51] H. Al-Jibbouri, I. Vidanović, A. Balaž, and A. Pelster, J. Phys. B 46, 065303 (2013).

[52] W. Kohn, Phys. Rev. 123, 1242 (1961).

[53] S. Stringari, Phys. Rev. Lett. 77, 2360 (1996).

[54] C. J. E. Straatsma, V. E. Colussi, M. J. Davis, D. S. Lobser, M. J. Holland, D. Z. Anderson, H. J. Lewandowski, and E. A. Cornell, Phys. Rev. A 94, 043640 (2016).

[55] Z. Shotan, O. Machtey, S. Kokkelmans, and L. Khaykovich, Phys. Rev. Lett. 113, 053202 (2014).

[56] A. Pricoupenko and D. S. Petrov, Phys. Rev. A 100, 042707 (2019).

[57] C. Chin, R. Grimm, P. Julienne, and E. Tiesinga, Rev. Mod. Phys. 82, 1225 (2010). 